\title{
La prueba de la eficiencia: un estudio de casos de las relaciones de colaboración que mantienen empresas con organizaciones sin fin de lucro en Chile'
}

\author{
DARÍO RODRÍGUEZ M.", RODRIGO FLORES GUERRERO", \\ PAULA MIRANDA SÁNCHEZ
}

\section{Resumen}

El propósito de este artículo es describir la relación de colaboración que mantienen cuatro empresas de capital español, con OSFL en Chile, en el marco de las acciones de Responsabilidad Social que desarrollan. Interesó observar el origen de la relación, la asignación de valor que las empresas atribuían a la relación y las posibles acciones o consecuencias, lecciones o aprendizajes que surgían de ellas.

Palabras claves: Relaciones de colaboración interorganizacionales. Organizaciones Sin Fines de Lucro. ONGS. Responsabilidad Social (RS).

\footnotetext{
${ }^{1}$ Este artículo da cuenta de algunos de los hallazgos encontrados en una investigación financiada por FONDECYT № 1100369.

* Doctor en Sociología. Profesor de la Facultad de Ingeniería de la Universidad Diego Portales. (Chile) E-mail: dariorodrigue@gmail.com.

** Doctor en Psicología Social. Profesor de la Facultad de Ciencias Sociales de la Pontificia Universidad Católica de Chile. (Chile) E-mail: rfloresu@uc.cl.

*** Doctora en Gestión Avanzada de Negocios Internacionales. Profesora de la Facultad de Ciencias Sociales de la Pontificia Universidad Católica de Chile. (Chile) E-mail: pmirands@uc.cl.
} 


\section{The proof of efficiency: a case study on cooperative relations between firms and nonprofits in Chile}

\section{Abstract}

The purpose of this paper is to describe the collaborative relationship established, within the framework of social responsibility initiatives, between four Spanish companies based in Chile and nonprofit Chilean organizations. The study focused on the origin of the relations, as well as the value assigned by the companies to such relationship and the possible consequences, lessons or learning they may produce.

Keywords: Inter-organizational collaborative relationships. Nonprofit organizations. NGO. Corporate Social Responsibility (CSR).

\section{Introducción}

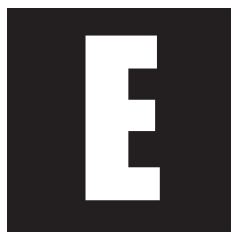

I propósito de este artículo es describir la relación de colaboración que mantienen cuatro empresas de capital español, con OSFL en Chile, en el marco de las acciones de Responsabilidad Social que desarrollan. La investigación estuvo interesada en observar el origen de estas relaciones de colaboración, la asignación de valor que las empresas atribuían a esta relación y las posibles acciones o consecuencias, lecciones o aprendizajes que surgían de ellas.

Tal como lo entendemos, el proceso de diferenciación que acompaña a la sociedad mundial nos lleva a observar la emergencia de diversos tipos de organizaciones y la relativa importancia que han ganado. En cuanto las organizaciones son sistemas artificialmente diseñados con el objeto de alcanzar racionalmente determinados fines, ellas tienen cabida en los sistemas de funciones de la sociedad, aunque la ejecución de las decisiones no se sustente en ellos. Estos sistemas sociales pueden nacer 
libremente, de modo que es posible observar innumerables diferenciaciones que no se relacionan con ninguno de los sistemas de funciones de la sociedad (Luhmann, 2007). Este es el caso del tercer sector, que busca enfrentar los problemas insuficientemente tratados por las organizaciones públicas gubernamentales y que velan por problemas dejados de lado por las organizaciones privadas con fines de lucro. Tales organizaciones se caracterizan por no ser dependientes del Estado ni perseguir fines de lucro y por eso se las puede denominar indistintamente Organizaciones No Gubernamentales (ONG) u Organizaciones Sin Fines de Lucro (OSFL) (Brinkerhoff, 2002, p. 19).

Definir las organizaciones en términos de la antigua teoría de sistemas abiertos (Emery; Trist, 1965; Katz; Kahn, 1966) hace difícil comprender que organizaciones con y sin fin de lucro puedan operar en el "mismo" entorno y no ayuda a encontrar vías de comunicación posibles entre ellas, principalmente porque hace ver que la búsqueda de financiamiento constituiría una actividad necesaria, pero perturbadora para las organizaciones sin fines de lucro, las cuales incluso podrían temer diluir su misión y erosionar su legitimidad al involucrarse demasiado en ella (Vaughan; Arsneault, 2003). No ayuda demasiado considerarlas a tal grado dependientes de quienes contribuyen a financiarlas, que deben dedicar parte importante de su ocupación a gestionar su independencia, ajustándose parcialmente a las demandas de sus financistas y tratando de cooptar o adquirir poderes del entorno para evitar el control por parte de éstos o buscando fuentes alternativas de financiamiento (Froelich, 1999, p. 247-248). Es evidente, no obstante, que esta perspectiva que considera a las OSFL dependientes de sus fuentes de financiamiento ha sido predominante, tanto entre los propios miembros de las OSFL, como entre los académicos interesados por estudiarlas que no disponen de una teoría diferente a la de sistemas abiertos (Pfeffer; Salancik, 1978). 
La teoría de la organización de Niklas Luhmann, permite superar este problema porque concibe a las organizaciones como sistemas autopoiéticos de decisiones que establecen límites de sentido con respecto al entorno. El sentido es una estrategia de selección entre posibilidades que no elimina las posibilidades desechadas, sino simplemente las deja suspendidas lo cual permite que en un momento posterior se seleccione alguna de esas posibilidades suspendidas, lo cual hace variar el sentido. Al ser autopoiéticos, los sistemas organizacionales quedan definidos como una red de producción de componentes que produce, con su operar, los componentes que la componen. Esto significa que las organizaciones operan clausuradamente, lo que les permite cierta autonomía respecto al entorno (Luhmann, 2011).

Aunque en la relación entre empresas privadas y OSFL, la forma filantrópica se presenta como la más frecuente (Austin, 2003, p. 44), desde fines del siglo XX ha aumentado la demanda, hecha a las OSFL por parte de sus donantes, de que mejoren su eficiencia en el uso de los recursos que les son otorgados (Koljatic; Silva, 2002, p. 5). La declinación del Estado de Bienestar ha implicado que las organizaciones del sector público restrinjan sus programas sociales, esperando que los servicios que ellas ofrecían sean asumidos por el tercer sector (Foster; Meinhard, 2002, p. 549-550). En pocas palabras, los rápidos cambios experimentados por la sociedad mundial han implicado un aumento de su complejidad, lo que ha hecho que las organizaciones de todo tipo, también las OSFL, hayan redefinido sus límites de sentido con respecto al entorno, volviendo a considerar posibilidades que alguna vez dejaran suspendidas. Parte importante de esta redefinición ha implicado volver sobre la distinción básica lucro/no lucro, que había sido entendida casi como rechazo moral al lucro, para descubrir que la negación que hace relevante el no lucro y deja el lucro como trasfondo irrelevante, permite ver un entorno bastante 
más amplio de posibilidades, excluyendo sólo el lucro como elemento diferenciador de la operación autopoiética de la organización. Considerando que la organización es operativamente clausurada, se minimiza el temor de contaminación que impediría a las OSFL establecer relaciones asociativas con organizaciones lucrativas. Se torna posible, por ejemplo, incorporar prácticas de administración y planificación estratégica que ayudan al mejor uso de los recursos y definir la misión como factor motivador (McDonald, 2007).

Las organizaciones lucrativas también han cambiado su perspectiva en la distinción lucro/no lucro. La indiferencia con que consideraban el lado del no lucro las hacía estimar de escaso interés relacionarse con organizaciones no lucrativas. Sin embargo, los cambios en las sensibilidades frente al medio ambiente, la creciente conectividad desarrollada por los individuos gracias a las nuevas tecnologías de la información, el aumento del poder de los clientes, el incremento de la capacidad de observación por parte de la sociedad mundial y la correspondiente exigencia de transparencia, que se efectúa a toda clase de organizaciones, han llevado a que las organizaciones con fines de lucro amplíen sus audiencias significativas, cambiando de la restringida preocupación por responder a sus shareholders a la necesidad de atender múltiples stakeholders (Porter; Kramer, 2011). Como consecuencia de esto, el lado del lucro se ha debido redefinir, para descubrir oportunidades de lucro en ámbitos antes desechados como no lucrativos. Este cambio de sentido es posible porque, como se ha dicho, el sentido no elimina las posibilidades descartadas, sino que sólo las suspende y puede, por lo tanto, seleccionarlas posteriormente (Luhmann, 2007). En los términos que nos interesan, esto ha significado que la relación con organizaciones no lucrativas que antes parecía irrelevante y se evitaba completamente o se restringía a los marcos de la filantropía, aparece bajo un prisma lucrativo que la hace ver interesante, 
por ejemplo, integrando una estrategia de marketing (Froelich, 1999, p. 252). Por otra parte, las influyentes ideas de Peter Drucker acerca de convertir la gestión en una profesión capaz de hacerse responsable por el bienestar y no sólo por la riqueza, agregando que la gestión era una vocación honorable si asumía responsabilidades más amplias, entre las que podía estar la colaboración con empresas no lucrativas, vuelven a ser recordadas por los especialistas (Kanter, 2009).

La colaboración entre empresas con afán de lucro y OSFL puede observarse, entonces, bajo un nuevo prisma. Las organizaciones con fines de lucro mejoran su imagen ante sus stakeholders, entre los cuales se debe destacar la comunidad en que están insertas, la opinión pública general, los clientes y también sus propios empleados, quienes muestran mayor disposición a comprometerse con una organización que apoya actividades altamente valoradas por la sociedad; este mejoramiento de la imagen contribuye, además, a atraer postulantes de mejor calidad y a enriquecer la cultura organizacional. Las OSFL no sólo consiguen mayor estabilidad para el flujo de sus recursos financieros, sino que adquieren los conocimientos de administración necesarios para hacer una mejor gestión de aquellos; la alianza les otorga, adicionalmente, el reconocimiento por parte del mundo económico que les abre nuevas oportunidades de acceso a fuentes de financiamiento $y$, con ello, mayor cobertura en el cumplimiento de su misión (Austin, 2000).

Junto a lo anterior, Stark ha desarrollado una distinción muy fructífera para la sociología económica. Se trata del concepto de valía, referido a lo valioso. La palabra inglesa Worth es aplicable tanto al valor moral como al económico. De acuerdo a esto, es posible concebir que en una organización y, con mayor razón en una alianza entre organizaciones, coincidan diferentes criterios para definir lo que es digno, lo que es valioso, lo que cuenta. Si bien la diversidad de criterios puede producir fricciones, en ella también se encuentra una gran capacidad de innovación (Stark, 2009, p. 6-8). 
La distinción valioso/no valioso incluye entonces criterios morales y económicos de evaluación, razón por la cual Stark (2009, p. 7) afirma que toda economía tiene un componente moral. Al utilizar esta distinción, se hace posible evitar la falsa concepción que hace de la creación de valor un asunto netamente económico, porque separa valor económico de valores morales:

[...] nos embarcamos en un análisis de lo valioso para desarrollar herramientas para entender un cálculo más rico que integra valor y valores, lo intelectual y lo emotivo, la valoración y lo evaluativo. Si vemos que los actos de estimación incluyen prácticas de estima, vemos que los sistemas de pago se refieren al reconocimiento tanto como a recompensas monetarias (Stark, 2010, p. 20).

Pueden coexistir múltiples órdenes de valía, cada uno de los cuales define lo bueno, lo justo y lo correcto desde sus propios criterios y el emprendimiento sería la capacidad de conjugar diversos criterios evaluativos y explotar la fricción resultante (Stark, 2010, p. 17). La fricción generada por la confluencia de criterios evaluativos diferentes no sería, por lo tanto, destructiva, sino creativa, proporcionando reflexividad organizacional al no imponer el consenso derivado de un único criterio de valoración, sino más bien promover una fricción productiva entre criterios de valía distintos.

Stark propone, además, el concepto de heterarquía para designar una forma organizacional de inteligencia distribuida en la cual las unidades colaboran de manera interdependiente de acuerdo a principios de evaluación diversos. Al no haber un orden jerárquico de dichos principios de evaluación, se produce entre ellos una fricción creativa. Aunque Stark utiliza el concepto para diseñar una nueva forma de empresa, este también resulta adecuado para comprender relaciones de beneficio mutuo entre organizaciones, porque las heterarquías crean riqueza al invitar más de una forma de evaluar lo valioso (Stark, 2010, p. 37). 
La propuesta de Stark contribuye a destacar la potencialidad de la colaboración entre organizaciones cuyos órdenes de valía son tan diversos como las empresas lucrativas y las OSFL. Las oportunidades abiertas por esta clase de alianzas heterárquicas van mucho más allá de los beneficios que se pueden conseguir mediante relaciones filantrópicas o transaccionales en la clasificación de Austin (2003, p. 47-51). La distinción valioso/no valioso implica, por lo tanto, diversos órdenes de valía. Esto significa que en la sociedad coexisten distintos modos de crear valor y que desconocerlo implica perder de vista importantes conocimientos que han sido ya demostrados, como la base misma del origen del capitalismo (Porter; Kramer, 2011). Como se sabe, a comienzos del siglo XX quedó fehacientemente documentado que la ética constituía un orden de valía aparentemente reñido con el afán de lucro y que, sin embargo, al ser perseguido con profunda convicción espiritual, condujo a la generación del valor económico que cimentó el surgimiento del capitalismo (Weber, 1984). En otros contextos espacio-temporales también se ha encontrado esta vinculación entre el orden de valía ético y la creación de valor económico (Martin, 1990). En los últimos tiempos, ha quedado en evidencia la importancia económica que pueden adquirir valores tales como la honradez, la confianza, la responsabilidad, el respeto al medio ambiente, la sociabilidad, el reconocimiento social, el conocimiento, etc. (Beinhocker; Davis; Mendonca, 2009, p. 62). No existe, por consiguiente, una necesaria oposición entre valor (económico) y valores (morales) (Stark, 2009). La observación de diferentes órdenes de valía, desde la perspectiva de sus posibilidades de generación de valor económico, ha dado origen a que se haya denominado "capital" a formaciones de valía no económicas, tales como capital humano (Davenport, 2006; Lawler, 2006), "capital intelectual (Stewart, 1998), capital social (Lin, 2001; Field, 2003) o capital cultural (Bourdieu, 1997). 
Considerar diversos órdenes de valía hace posible comprender que todas las organizaciones buscan crear valor, aunque lo hagan desde distintos órdenes de valía. Aceptar que toda economía es un orden moral o que órdenes de valía diferentes generan valores que pueden ser también económicamente valorados conduce a facilitar el diálogo entre organizaciones que no sigan los mismos principios. Reconocer que toda organización, también la sin fines lucrativos (OSFL), ha de gestionar eficientemente sus recursos y hacer coincidir su estructura con su misión (Chandler, 1962; Drucker, 1992), obliga a las OSFL a abandonar el estilo asistencial-paternalista que caracterizara a muchas de ellas durante gran parte del siglo XX. Todo esto habla a favor de la colaboración y la alianza entre OSFL y empresas con ánimo lucrativo.

Tal como se ha dicho, la sociedad es un sistema social único que, por lo mismo, tiene carácter mundial. Para las organizaciones insertas en la sociedad mundial, esto significa que es preciso responder a las exigencias de stakeholders muy lejanos, pertenecientes a otros hemisferios y cuyas sensibilidades pueden ser diversas a las que tienen personas pertenecientes a la misma región. A modo de ejemplo, la Responsabilidad Social (RS) exigida a toda clase de organizaciones incrementa continuamente su ámbito de consideración (Zadek, 2007). A la protección del medioambiente, se agregan las condiciones laborales, la manipulación de los alimentos, el trato a los animales, el uso de sustancias químicas, la sensibilidad a los problemas sociales, etc. Las capacidades de observación y la interconectividad también mejoran, haciendo que sea cada vez más importante cumplir con las condiciones de RS, para evitar problemas derivados del escrutinio y la correspondiente respuesta coordinada de grupos inesperados de stakeholders. Todos estos ámbitos de interés fueron atribuidos rápidamente a la capacidad de respuesta y solución que las OSFL podían ofrecer (Bradach; Tierney; Stone, 2009) 
Es un hecho conocido que órdenes de valía o valores no económicos son determinantes para la conducción exitosa de los negocios. Si bien resultan difíciles de cuantificar en términos monetarios, muchas veces su estimación alcanza cantidades impresionantes. Después de los escándalos de corrupción en importantes organizaciones del ámbito financiero y del retail: ¿Cuánto vale una reputación intachable? Después del repudio de los jóvenes europeos y estadounidenses a Nike, por haber subcontratado la producción de algunos de sus artículos a fábricas que acordaban, legalmente, contar con mano de obra infantil (Zadek, 2005): ¿Cuánto vale el respeto a los derechos de la infancia, en la sociedad mundial? Después de la muerte de los cisnes del río Cruces, en el Sur de Chile: ¿Cuánto vale la sensibilidad ciudadana?

Por su parte, las OSFL también se ven confrontadas por el escrutinio de la sociedad. Se les exige eficiencia y uso ético y responsable de sus recursos. Deben poder demostrar que contribuyen efectivamente a la solución del problema social que las inspira. La transparencia, en este caso, se refiere a contabilidades rigurosas y la estricta vigilancia de sus modos de operación. Para ello pueden aprender mucho de las organizaciones lucrativas. Si se entiende que también ellas generan valor, es preciso resguardar que esa generación de valor no se corrompa, que el dinero recibido no se filtre inadvertidamente, que los responsables por la protección de las especies animales o vegetales no se involucren en su explotación, etc. También las OSFL tienen una reputación que deben cuidar y no basta con la dedicación a una buena causa para tener la reputación asegurada; además deben demostrar que realizan su cometido con el mejor nivel de excelencia tanto ético como económico. 


\section{Metodología}

Teniendo presente los objetivos de la investigación, se optó por una metodología cualitativa, que combinara el estudio de casos con la utilización de grounded theory. La elección de la muestra y definición de variables se ciñen a los principios del estudio de casos en tanto su análisis sigue los principios de la grounded theory. Estas dos metodologías son adecuadas cuando se pretende contribuir a la descripción de fenómenos poco analizados (Harrison; Freeman, 1999), como es el caso de alianzas de colaboración entre empresas y OSFL en Chile.

Siguiendo a Eisenhardt (1989), se formuló una lista de variables que abordaron cada uno de los tres puntos indicados para nuestra investigación y se construyó un guión de entrevista semi-estructurada. Considerando el trabajo previo realizado en Chile por Koljatic y Silva (2002), las dimensiones a explorar en estas entrevistas fueron: a) orígenes de la relación (por qué surgió); b) características de la relación (recursos aportados, participación de los socios, riesgos asumidos, entre otras); c) asignación de valor (valor social y económico, tiempo dedicado a mantener la relación, etc.) y d) factores claves (dificultades, impacto percibido, aprendizajes, grado de contacto/comunicación, etc.).

La muestra quedó conformada por los encargados o responsables de llevar la RSE y alianzas de colaboración con OSFL orientadas a Servicios Humanos, de cuatro empresas de capital español con presencia en Chile. A partir de ello, se siguieron los principios del muestro teórico (Glasser, 1978; Strauss; Corbin, 2002). Los casos fueron elegidos pues nos permitían aprender sobre los temas objeto de estudio, no por su representatividad estadística, tamaño, sector (Locke, 2001). La selección muestral se realizó a partir de una serie de criterios razonables, y equilibrando el principio de igualdad con el de diferencia (Flores, 2009; Locke, 2001). El principio de igualdad está determinado por los objetivos del estudio; 
se consigue por el origen de las empresas (españolas con operaciones en Chile) y las relaciones que mantienen con 9 organizaciones no lucrativas (mantienen relaciones de tipo económico con empresas y su misión es de tipo humanitario). Dentro de este grupo, el principio de diferencia se consigue porque las organizaciones elegidas son de distinta industria y tamaño. Las empresas seleccionadas pertenecen al rubro financiero, telecomunicaciones y energía.

A los encargados de RSE les solicitamos que nos indicaran las alianzas de colaboración que ellos identificaban como significativas. Además, se seleccionaron las OSFL's con las que mantienen relación, mediante la consulta de los informes de Responsabilidad Social y las páginas web. Tomamos contacto con los responsables de las alianzas de colaboración por parte de las OSFL's a los cuales les aplicamos una entrevista semi-estructurada que exploraba las variables de la investigación, tal como lo proponen los manuales de metodología cualitativa de investigación (Valles, 2000; Flores, 2009). La muestra final quedó conformada de la siguiente manera (Tabla 1):

Tabla 1

\begin{tabular}{c|c}
\hline Empresas & OSFL relacionada \\
\hline
\end{tabular}

OSFL1: Rehabilitación de adicciones

OSFL2: Educación sectores vulnerables

E1: Empresa rubro financiero, Banco

OSFL3: Erradicación de campamentos OSFL4: Pobreza y exclusión social

\begin{tabular}{l|l} 
& OSFL4: Pobreza y exclusión social \\
\hline E2: Empresa de telecomunicaciones & OSFL5: Integración de discapacitados \\
OSFL6: Voluntariado internacional & OSFL7: Pobreza y exclusión social
\end{tabular}

\section{E3: Empresa de Energía hidroeléctrica}

E4: Empresa de Energía explotación de gas

OSFL8: Inclusión de pueblos originarios OSFL9: Pobreza y exclusión social
OSFL10: Museo

OSFL11:Fomento educación técnico-profesional OSFL12: Pobreza y exclusión social 
Las entrevistas fueron grabadas guardando recaudo del consentimiento informado y cada una de ellas fue transcrita inmediatamente y analizada antes de realizar la siguiente, codificando línea por línea. Estos códigos fueron agrupados en categorías, empleando el paradigma de codificación axial establecido por Strauss (1987). También se siguieron las sugerencias realizadas por los autores de referencia en diseño de casos (Eisenhardt, 1989): seleccionar pares de casos y listar las similitudes y diferencias entre cada par. Para determinar la fiabilidad de los resultados seguimos las sugerencias establecidas por Strauss y Corbin (2002, p. 253): consistencia entre codificadores y exposición de los resultados a juicio de expertos.

\section{Principales resultados}

Siguiendo las directrices impartidas por grounded theory (Glasser, 1978; Strauss; Corbin, 2002), para el análisis de las entrevistas realizadas, la categoría central de la investigación es la colaboración como generador de valor. En términos genéricos las empresas reconocen que las alianzas responden a una estrategia mayor que tiende a ligarse con la generación de valor social y económico. Desde ahí, señalan como características de tales estrategias el que ella se guía por: mejorías en la cadena de valor; medición de los impactos que la RS logra; relevancia del marketing. De esta manera, tanto los estudios como la publicación y los reportes anuales de RS son factores que favorecen la generación de valor en las empresas.

Junto a ello, se establece que los modos de medición aún son insuficientes en el país. Las empresas reconocen que las alianzas generan valor provenientes de una mejoría en la reputación corporativa, la que a su vez es consecuencia de la macro-estrategia de RS. Entre los valores mencionados se cuentan: los accionarios, de calidad de RRHH, de mejoría de la imagen ante los clientes, entre otros. 
Imagen 1 -

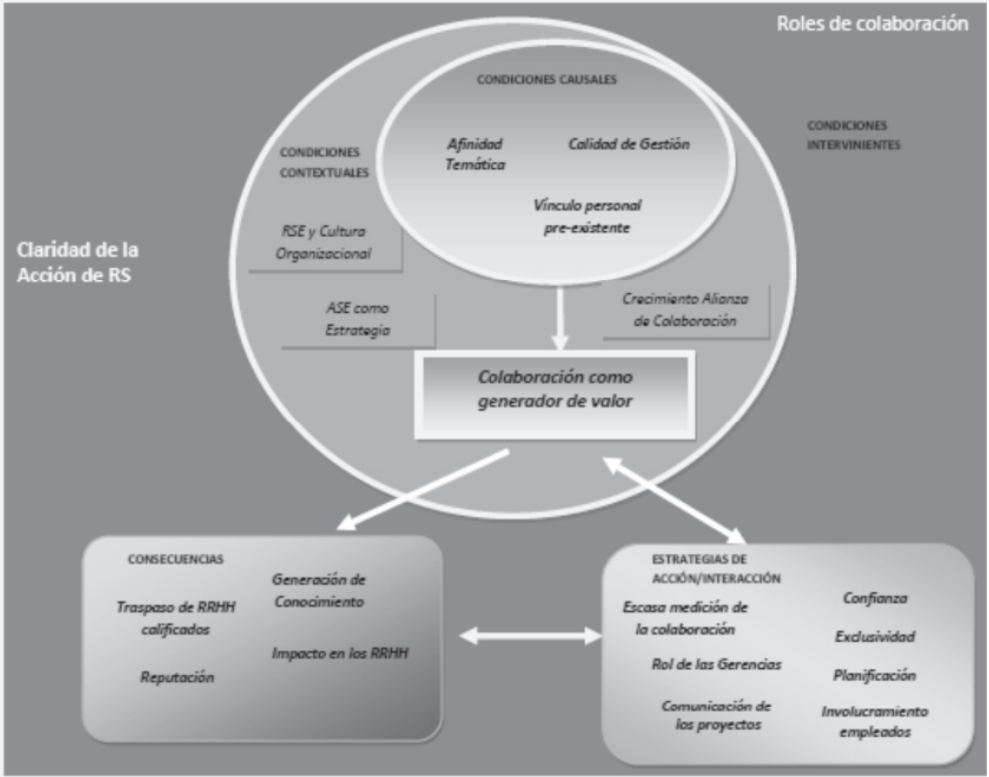

Fuente: Elaboración de los autores

\subsection{Condiciones contextuales}

Entendemos por condiciones contextuales a aquellos elementos específicos que se entrecruzan en un tiempo y lugar para crear un conjunto de circunstancias o problemas a los cuales responden las personas en las acciones/ interacciones (Strauss; Corbin, 2002, p. 144). Para el estudio de casos de esta investigación, reconocemos 3 elementos que cumplen tal condición:

1. Responsabilidad Social Empresarial en sintonía con la cultura interna. Ello implica por lo menos tres áreas de interés: a) el desarrollo de una estrategia de RS con un involucramiento activo de los empleados ("en 
nuestro caso, tenemos el "Uno más Uno", involucramos a nuestros empleados"); b) solicitar espacios físicos y dotar del tiempo necesario para que los trabajadores puedan desarrollar su responsabilidad social como expresión de la cultura corporativa que demanda nuevas formas de implicación con la sociedad ("a lo mejor 10 años atrás era impensado, o sea, cada uno apoyaba a través de sus familias, su iglesia, pero ya te exigen los empleados cuando la empresa da esta posibilidad...."); y c) la profesionalización e institucionalización al interior de la empresa del voluntariado corporativo ("Tienen un presupuesto asignado, se trabaja como cualquier proyecto");

2. Acción social forma parte de la estrategia. Es posible observar que no existe una idea compartida entre las empresas estudiadas, sobre cuál es la visión global en la cual se encuentran insertas las alianzas. Sin embargo, se entiende que las alianzas forman parte de una estrategia de Acción Social Empresarial (ASE) mayor y planificada que incluye inversión social, generalmente filantrópica, del tipo donación en dinero, muy instaurada en Chile. Del mismo modo, se indica que se prefiere invertir más en RS que publicitar sus acciones entendidas solamente como una acción de marketing ("no vamos a estar gastando unos millones en publicitar algo social, si esos mismos los podríamos estar gastando en el mismo programa");

3. Crecimiento y desarrollo de la alianza. Un elemento que surge a partir del análisis de las entrevistas dice relación con las características propias que asumen las alianzas. Ello supone un fuerte involucramiento para la gestión de la empresa, que va incrementándose con el transcurrir del tiempo. Es habitual que la relación parta lentamente de forma filantrópica y vaya madurando hacia formas de mayor afectación mutua ("la relación nuestra partió con el cheque y ahora va mucho más de gestión y de involucrarnos en la fundación"). 


\subsection{Antecedentes o causas}

Entendemos por condiciones causales, aquellos elementos que suelen representar conjuntos de acontecimientos que influyen de manera directa sobre el fenómeno de estudio (Strauss; Corbin, 2002). De acuerdo a las entrevistas realizadas podemos indicar que las condiciones causales de las relaciones de colaboración basadas en la confianza son tres:

1. Afinidad temática. El criterio principal para establecer alianzas de colaboración es la afinidad con los temas que trabaja la empresa, de manera que aquellas OSFL que posean otros intereses o ámbitos de acción no estarían siendo consideradas ("yo ahí hice un mapeo, casi un casting, hice un mapeo completo de todas las ONG que trabajaran con niñez, que trabajaran con el tema educación") Este elemento de selección nada dice sobre la complejidad que pueda asumir el vínculo en el futuro. En muchos casos, las alianzas poseen mucho de "ir probando" con el transcurrir del tiempo, si los programas y proyectos propuestos permiten ir aumentando su oferta y cobertura, tanto es así que las empresas entrevistadas indican que la concreción de las alianzas es un proceso de "madurez", que requiere "tiempo" y "profundidad", la mayoría de las veces, incremental. ("En general con todas las fundaciones, ha sido un continuo, un avanzar");

2. Calidad de gestión reconocida. Un segundo criterio para que surja una colaboración es que la OSFL haya demostrado poseer una calidad reconocida en su gestión, ello pasa por el tamaño de la organización y por el impacto de sus productos ("Yo creo que estar bien gestionado") También se nombra la importancia de realizar una actividad en forma profesional, lo cual ayudaría a reforzar la confianza ("el tema de la seriedad, por lo menos para mí, la seriedad, el profesionalismo y las confianzas"); 
3. Vínculo personal pre-existente. Un tercer criterio en el establecimiento de las alianzas corresponde a un vínculo personal preexistente entre quienes establecerán la colaboración. Este vínculo personal pasa siempre por un conocimiento previo de la OSFL por su experiencia o reconocimiento, o por una cercanía de amistad establecida con anterioridad entre los directivos de ambas organizaciones ("no hay contrato de por medio, no hay nada, son alianzas que están basadas en las personas, en compromisos, ite fijas o no?, y las personas que están ahí detrás son fundamentales para el desarrollo del proyecto; sí, yo te diría que son las personas"). Este elemento parece ser fundamental no solo para la selección de las OSFL sino también para la mantención e incremento del vínculo en el tiempo.

\subsection{Condiciones intervinientes}

Entenderemos por condiciones intervinientes a aquellos elementos que mitigan o alteran el impacto de las condiciones causales sobre los fenómenos (Strauss; Corbin, 2002). De acuerdo a nuestros registros es posible identificar en esta categoría dos tipos:

1. Claridad de la RSE. En términos generales, la mayoría de las empresas entrevistadas posee una claridad en la línea de RSE a desarrollar, especializándose en ámbitos de acción específicos. Así por ejemplo, algunas empresas han asumido temas específicos para abordar necesidades de infancia y trabajo infantil, otra tiene un vínculo estrecho con una fundación que tiene un vínculo profundo con las comunidades en las que ejerce una acción directa y una tercera tiene vínculos con una OSFL que realiza acciones en educación y cultura. Sólo una empresa parece no poseer un foco de acción concentrado en un solo ámbito, abordando variados elementos al mismo tiempo. 
2. Diferentes roles de las empresas asumidos en las alianzas de colaboración, de los cuales identificamos por lo menos tres:

Rol de financistas de las acciones sociales y programas que llevan adelante las OSFL ("el compromiso es que la empresa se compromete al financiamiento de la Fundación con un monto anual, en estimador UF (Unidad de Fomento) ${ }^{2}$ que ha ido cambiando en el tiempo, que creo que actualmente son 9 mil UF anuales más el 0,3 \% de las utilidades").

Rol publicitario, donde por medio de las acciones sociales ejercidas por la empresa se da un espacio de comunicación y publicidad a la OSFL ("Eso ellas lo valoran. Y que ellos no podrían hacer esa difusión, pero uno como empresa si se las puede hacer ite fijas o no?"), lo cual incluye el ejercicio del marketing social ("campaña de marketing social, que es donde ponemos toda la red de sucursales, todos los ejecutivos comerciales, para captar recursos").

Rol facilitador. Además de todo lo anterior, se espera que las empresas asuman un rol facilitador de diversas acciones que permitan mantener en funcionamiento la ASE, por ejemplo, el que sus trabajadores desarrollen voluntariados corporativos en las OSFL ("hay grupos de voluntarios que apadrinan durante el año alguno de los jardines infantiles de la Fundación").

\subsection{Consecuencias}

Por consecuencias entendemos aquellos elementos de causalidad que se presentan toda vez que el fenómeno se manifiesta (Strauss; Corbin, 2002). De acuerdo a nuestra investigación es posible identificar las siguientes:

\footnotetext{
${ }^{2}$ En Chile 1 Unidad de Fomento equivale a aproximadamente 43 dólares americanos.
} 
1. Traspaso de recursos humanos calificados. La conformación de las alianzas de colaboración permite un flujo de recursos humanos desde la empresa hacia la OSFL, enriqueciendo de esta manera a la sociedad civil. Ello se expresa de dos maneras, como voluntariado corporativo, restringido a la ciudad de Santiago y sus alrededores, o como el involucramiento de ejecutivos de primera línea en los directorios de fundaciones o corporaciones ("en el caso de la educación, de la fundación, Francisco [], que hoy día es director... está a cargo de toda el área comercial, o sea, un ejecutivo de primera línea, es director de la fundación“)

2. Generación de conocimiento. Un tema abordado con las empresas entrevistadas fue la generación de conocimientos o buenas prácticas que hayan surgido como instancias del desarrollo de las alianzas de colaboración. Se reconoce que las alianzas constituyen instancias para vincular a la empresa con la realidad del mundo social. También se entiende como la posibilidad de abordar de mejor manera clientes comerciales antes no percibidos, cubiertos o descuidados ("hacemos cursos de capacitación a microempresarios. Se trabaja mucho con las municipalidades") Este es un elemento importante, pues se indica que las empresas saben de gestión, poseen voluntariado corporativo y visión de negocio, activos que se transfieren a las organizaciones no lucrativas ("traspasar gestión a las fundaciones, que muchas veces eso, en un principio las fundaciones no lo ven, pero después ven que... que los ojos empresariales la pueden ayudar mucho más que en dar los recursos")

3. Reputación como consecuencia de la RS. Se considera que la reputación empresarial es una consecuencia y no una finalidad de la RS efectuada por la empresa. La reputación queda entendida como algo que se logra producto de un trabajo, que lleva tiempo conseguirla y debe estar vinculada a la sustentabilidad ("si se cree que va a llegar a la reputación por la responsabilidad social solo así, no, porque es de corto plazo"). 
Esta idea de reputación, se entiende como generadora de valor económico producto de la relación, aunque la expresión de ese valor surge más bien como un ideal, como deseabilidad social, más que como un elemento efectivo y tangible que pueda ser medido o que pueda ser atribuido exclusivamente a una relación de colaboración en particular con una OSFL ("en el ámbito social y público nos conocen, los colegios nos llaman y nos dicen: "queremos entrar al programa")

4. Impacto en los RRHH. Las empresas entrevistadas nos indicaron que las alianzas de colaboración planteaban claras implicancias en el desarrollo de los procesos de Recursos Humanos. Ello se expresa de dos maneras: a) como atracción de nuevos profesionales y b) como una mejora concreta en las tasas de contratación, aunque ninguna de las empresas entrevistadas posee indicadores de medición tangibles para avalar dicha afirmación. Otras consecuencias en la gestión de RRHH nombradas por las empresas son: a) mejora en el clima laboral producto de saber que se realiza una serie de acciones sobre la sociedad; b) mayor sentido de pertenencia e implicación de los trabajadores con las acciones realizadas por la empresa; c) mejora en el trato directo y atención de los clientes; y d) bajas tasas de rotación a raíz de la ASE, no obstante estos cuatro elementos no se encuentran medidos por medio de correlaciones directas entre las acciones ejercidas por la ASE y los indicadores que los departamentos de RRHH registran.

\subsection{Estrategias de acción/interacción}

Por estrategias de acción/interacción entenderemos aquellas tácticas o rutinas o el cómo maneja la gente sus problemas y asuntos con los que se encuentra en forma cotidiana cuando hace frente al fenómeno. Son actos deliberados o ejecutados a propósito para resolver un problema y al hacerlo moldean el fenómeno (Strauss; Corbin, 2002). 
1. Rol Asumido por las de Gerencias. Las empresas entrevistadas nos indicaron que existe un involucramiento activo de las gerencias en la conformación y mantención de las alianzas de colaboración con las OSFL. Ello se expresa como una inserción activa de los gerentes en las actividades y directorios de las OSFL como consecuencia de esa relación específica, lo que se observa como producto del desarrollo de una confianza entre ambas organizaciones ("Yo diría que el involucramiento corporativo también es un gesto de confianza para la fundación“). Otro elemento importante del rol activo que asumen las gerencias se expresa en la necesidad de evaluar las actividades desarrolladas en conjunto, por medio de los programas de acción ("este plan es evaluado por la primera línea de gerentes y el gerente general dos veces al año"), lo que implica que necesariamente la ASE se encuentra integrada a la estrategia de la empresa ("cómo nos evaluamos, cómo monitoreamos, nosotros cumplimos un rol de monitoreo muy fuerte, de ver que el resto de las gerencias y las áreas estén cumpliendo y estén llevando a cabo las metas que tiene la sostenibilidad, se desarrolla un plan anual ")

2. Comunicación en el desarrollo de los proyectos. Para las empresas entrevistadas mantener una comunicación fluida y bidireccional que permita el desarrollo adecuado de los proyectos y programas ejecutados pasa a ser un elemento fundamental. En función del reconocimiento de la relevancia de la implicación del público interno en la estrategia de RS es que las empresas no sólo comunican hacia la sociedad los logros de dicha colaboración, sino que también lo hacen en dirección a sus propios miembros, buscando así una validación interna, además de un impacto positivo en dimensiones que involucran la pertenencia, el clima laboral, la tasa de rotación, entre otros. Las estrategias de comunicación que se desarrollan en las alianzas de colaboración son los siguientes: a) Exposición de las ASE. Las empresas indican que habitualmente realizan 
exposiciones anuales de las acciones sociales a distintos públicos externos con los cuales se encuentran involucradas ("nosotros tenemos un reporte social que lo mostramos una vez al año"); b) Publicidad interna de ASE. Otras empresas realizan en forma periódica una comunicación de los resultados de las alianzas de colaboración con su público interno ("en general el área de sostenibilidad se ha preocupado de que los trabajadores conozcan y estén al tanto, y toda la organización esté al tanto, de lo que hace la empresa")

3. Confianzas instauradas. Otro elemento relevante dice relación con el desarrollo y permanencia de confianza entre ambas partes, en realizar un trabajo adecuado y profesional. La confianza se utiliza para instaurar la relación de colaboración y para mantenerla en el tiempo. También se observa en el incremento de la relación, en el desarrollo de nuevos programas a abordar en conjunto y al pasar a ser algo más que tan sólo filantropía ("uno va profundizando en la medida que va teniendo confianza con los equipos, con quienes los dirigen, cómo actúan como fundación frente a la sociedad").

4. Escasa medición de las alianzas. Las empresas analizadas indican que el desarrollo de la RS en Chile es un proceso que aún no permite generar mediciones constantes sobre las acciones realizadas, de manera que no se mide la consecución de los objetivos de la ASE ("cuando tú haces una consulta a tus recursos humanos sobre, no sé, por ejemplo: “¿cómo coordinas que la relación con las comunidades se va a cruzar por aspectos internos?, es muy normal andar escuchando discursos como: "iah!, estás regalando dinero pero no mejoras los beneficios", entonces siempre, es muy difícil la medición"). Ello lleva asociado el hecho de un escaso interés por establecer metodologías e indicadores que ayuden a monitorear las acciones realizadas. Entre los argumentos que se indican para explicar este fenómeno, se señala que es muy difícil separar variables que 
tienden a ser multicausales ("El problema es que, metodológicamente, es muy difícil separar la gestión de la sostenibilidad o de la responsabilidad social de muchos otros factores"), lo que impide también vincularlos a otros elementos, como por ejemplo, su incidencia en la reputación corporativa. No es extraño que la ausencia de metodologías e indicadores tenga una incidencia directa en la ASE desarrollada por las empresas, ya que si no se puede medir el logro del trabajo de las OSFL, la inversión de recursos no pasará a ser considerada más que filantropía.

5. Involucramiento de los empleados. Las empresas entrevistadas reconocen que sus empleados poseen una participación en la implementación de acciones de RSE, lo que implica un involucramiento activo en las estrategias de ASE ("Preguntarles, hacerlos partícipes, ponte tú, cuando hacemos la memoria de responsabilidad corporativa, que también tenemos diálogo, incorporamos los sindicatos, los empleados, les preguntamos: "¿qué les parece lo que estamos publicando?, ¿están de acuerdo? o ¿cuáles son sus contribuciones?, ¿qué creen que deberíamos poner?", y después, en la memoria siguiente tú les das respuesta a esas inquietudes que plantean").

6. Planificación en conjunto. Las empresas entrevistadas indican que una de las características de los proyectos realizados en conjunto con las OSFL tiene que ver con una planificación activa de ambas partes, donde hay un mutuo involucramiento ("la directora que tiene que ver... de relaciones institucionales, se involucra en los contenidos del libro, o sea... hay reuniones aquí semanales"). Ello conlleva la exigencia de establecer relaciones del tipo exclusivo entre la OSFL y la empresa. Se les estaría solicitando a las OSFL que mantengan una relación única que impediría realizar acciones de colaboración o aceptar donaciones con otras empresas ("nosotros somos la empresa de la Fundación X"). 


\section{Conclusiones}

La colaboración entre empresas y OSFL en América Latina es aún poco conocida. No existe una gran variedad de estudios regionales que arrojen cifras sobre los montos de inversión social de las empresas o de sus características históricas. Los datos recopilados por Sanborn (2005), Austin, Gutiérrez, Ogliastri y Reficco (2006), Koljatic y Silva (2002) al igual que nuestra evidencia, nos indican que muchas de ellas pueden ser consideradas como "híbridas", es decir combinan donaciones en dinero con la realización de proyectos y programas propios. Ello puede deberse a una característica derivada de una mezcla de tradiciones filantrópicas latinoamericanas y, en ciertos casos, puede constituir una ventaja comparativa y una forma más adecuada para agregar valor.

Un elemento interesante de destacar es que las relaciones de colaboración desarrolladas en nuestros casos, se basan en atributos culturales (Rodríguez; Quezada 2007), principalmente en nexos interpersonales ya establecidos, centrados en la confianza (amistad o relación de parentesco). En este contexto, la colaboración es entendida como incremental, pues debe desarrollarse paso a paso, sorteando los obstáculos de la desconfianza y avalada en una buena gestión de los recursos. En este sentido, depende mucho de la atención y energía que brindaban los líderes de las empresas.

Tal como lo indica la literatura, las alianzas de colaboración entre empresas y OSFL pareciera que sólo pueden darse en ambientes de una marcada estrategia de RS. La evidencia parece demostrar que estas alianzas pueden entregar ventajas competitivas de importancia, mejorando la elección de los consumidores por la adaptación de bienes y servicios a las necesidades de los segmentos no rentables (Brinkerhoff, 2003), ofrecer mejores rendimientos para los inversores que responden a consideraciones de bienestar social y promover una mayor eficiencia del mercado por la competencia, exponiendo las acciones contrarias ella. A pesar de los 
diferentes objetivos de ambos sistemas sociales, los estilos, las misiones, y las culturas, las organizaciones, de distinto tipo, pueden colaborar para beneficio común (Lindenberg, 2001).

La mayoría de los casos de colaboración reseñados en esta investigación incluyen expresiones de filantropía y donación de dinero, no puede dejar de mencionarse importantes relaciones de transacción, al involucrarse en programas y proyectos que perduran a lo largo del tiempo. Bajo esta última dimensión, las OSFL son vistas como una fuente valiosa de conocimientos y experiencia sobre las cuestiones sociales y ambientales, y como más accesibles y confiables que el Estado (Austin, 2000; Austin; Gutiérrez; Ogliastri; Reficco, 2006). La experiencia nacional e internacional releva el hecho de que la conducción socialmente responsable de las empresas, genera decisiones de negocios mejor informadas, construye fidelidad, enriquece la imagen corporativa y de marca y contribuye de manera cuantificable a una rentabilidad corporativa de largo plazo. Además, cada vez adquiere mayor importancia el hecho de que los clientes y la sociedad en general, esperan y exigen que las empresas jueguen un rol protagónico en el desarrollo económico, en el cuidado del medioambiente $y$ en el desarrollo social de las comunidades de la cual forman parte (Porter; Kramer, 2011; Zadek, 2007; Post; Lee; Preston; Sach, 2002).

De acuerdo a la literatura, las OSFL reciben, como beneficio de la alianza, recursos financieros, servicios, conocimiento y tecnologías, acceso a otras corporaciones, mayor reconocimiento de marca y nuevas perspectivas (Austin, 2000). Las empresas, por su parte, son objeto de mejoras en su imagen, optimizan su reputación entre los empleados, reclutan candidatos de mayor calidad, incrementan su retención de personal, desarrollan habilidades, renuevan productos o servicios, logran aumentar los niveles de apoyo de los consumidores y su participación de mercado y enriquecen la cultura y valores organizacionales (Austin, 2000; Austin; Gutiérrez; Ogliastri; Reficco, 2006). 
Un elemento importante de la colaboración que establecen las empresas con OSFL es la escasa medición de las acciones realizadas. La evidencia encontrada nos muestra que las empresas no disponen de indicadores capaces de observar el impacto de la contribución realizada tanto a la propia OSFL como a la misma empresa. Este es un elemento que puede tener su explicación en la marcada filantropía con que es asumida la relación, lo que volvería innecesario desarrollar elementos de gestión. La literatura internacional indica que tales alianzas pueden tener un impacto intangible en la medición del desempeño financiero de la compañía, siendo su contribución no negativa, es decir, al menos no es dañina y existen razones para pensar que es positiva en términos financieros (Tsoustsoura, 2004; Ruf et al., 2001), aunque los estudios realizados no son concluyentes sobre esta materia.

Tomando en consideración las limitaciones de nuestra muestra, podemos afirmar que las tres etapas del continuo propuesto por Austin (2000) parecen ser más bien una tipología ideal y no una vía probable de evolución de esta clase de alianzas. El continuo evolutivo de las alianzas de colaboración entre empresas lucrativas y OSFL propuesto por Austin (2000) se basa fundamentalmente en la dinámica de evolución propia del proceso progresivo de colaboración. No toma en consideración la incidencia de factores del entorno de las alianzas, sean estos de índole cultural, política, legal, social o incluso económica. No se logra comprender con ese enfoque que las empresas no demuestren mayor interés en mantener la colaboración con las OSFL en el largo plazo. Las leyes que incentivan el aporte privado a la solución de problemas sociales, como la que permite deducir donaciones de impuestos, tampoco son consideradas por el modelo de Austin. Las crecientes demandas ciudadanas en contra del daño ambiental y social causado por empresas o incluso proyectos de empresas pasan desapercibidas por un modelo cerrado de evolución como el de Austin. Todos estos factores del entorno de las alianzas de colaboración, sin embargo, 
permiten comprender las dificultades que han tenido para desarrollarse en Chile y pronosticar el surgimiento de nuevas formas de colaboración que nunca tuvieron un origen meramente filantrópico.

\section{Referências}

AUSTIN, James et al. Un proyecto de investigación colectiva de la SEKN. México: Planeta, 2006.

AUSTIN, James. El desafío de la colaboración. Buenos Aires: Granica, 2003.

AUSTIN, James. Strategic Collaboration between Nonprofits and Business. Nonprofit and Voluntary Sector Quarterly, v. 29, n. 1, suppl. 1, p. 69-97, marzo 2000.

BEINHOCKER, Eric; DAVIS, Ian; MENDONÇA, Lenny. Las 10 tendencias que usted debe observar. Harvard Business Review, v. 87, n. 7, p. 60-65, jul. 2009.

BOURDIEU, Pierre. Capital cultural, escuela y espacio social. México: Siglo Veintiuno, 1997.

BRADACH, Jeffrey; TIERNEY, Thomas; STONE, Nan. Cumplir la promesa de las organizaciones sin fines de lucro. Harvard Business Review, v. 87, n. 8, p. 42-52, agosto 2009.

BRINKERHOFF, Jennifer. Government-Nonprofit Partnership: a Defining Framework. Public Administration and Development, v. 22, n. 1, p. 19-30, marzo 2002.

CHANDLER, Alfred. Strategy and structure: Chapters in the history of the industrial enterprise. Cambridge, MA: MIT Press, 1962.

DAVENPORT, Thomas. Capital humano. Barcelona: Deusto, 2006.

DRUCKER, Peter. Managing the Non-Profit Organization. New York: Harper Business, 1992.

EISENHARDT, Kathleen. Building Theories from Case Study Research. Academy of Management Review, v. 14, n. 4, p. 532-550, 1989.

EMERY, Frederick; TRIST, Eric. The Causal Texture of Organizational Environments. Human Relations, v. 18, n. 1, p. 21-31, feb. 1965.

FIELD, John. 2003. Social capital. London: Routledge.

FLORES, Rodrigo. Observando observadores: una introducción a las técnicas cualitativas de investigación social. Santiago: Ediciones Universidad Católica, 2009 . 
FOSTER, Mary; MEINHARD, Agnes. A Regression Model Explaining Predisposition to Collaborate. Nonprofit and Voluntary Sector Quarterly, v. 31, n. 4, p. 549-564, dic. 2002.

FROELICH, Karen. Diversification of Revenue Strategies: Evolving Resource Dependence in Nonprofit Organizations. Nonprofit and Voluntary Sector Quarterly, v. 28, n. 3, p. 246-268, sept. 1999

GLASSER, Barney. Theoretical Sensitivity: Advances in the Methodology of Grounded Theory. Mill Valley, CA:University of California, 1978.

HARRISON, Jeffrey; FREEMAN, R. Edward. Stakeholders, Social Responsibility, and Performance: Empirical Evidence and Theoretical Perspectives. Academy of Management Journal, v. 42, n. 5, p. 479-485, 1999.

KANTER, Rosabeth. Lo que nos diría Peter: la permanente relevancia de la perspectiva de Drucker. Harvard Business Review, v. 87, n. 11, p. 57-63, nov. 2009. KATZ, Daniel; KAHN, Robert. The social psychology of organizations. New York: John Wiley \& Sons, 1966.

KOLJATIC, Mladen; SILVA, Mónica. Variables asociadas a experiencias de colaboración entre empresas con y sin fines de lucro: Un estudio de casos. Revista Abante, v. 1, n. 5, 2002.

LAWLER, Edward. Por fin ha llegado la era del capital humano. In: BENNIS, Warren; SPREITZER, Gretchen; CUMMINGS, Thomas. Las claves del liderazgo. Barcelona: Deusto, 2006. p. 26-38.

LIN, Nan. Social capital: a theory of social structure and action. Cambridge: Cambridge University Press, 2001.

LOCKE, Karen. Grounded Theory in Management Research. California: Sage, 2001.

LUHMANN, Niklas. La sociedad de la sociedad. México: Herder, 2007.

LUHMANN, Niklas. Organización y decisión. México: Herder, 2011.

MARTIN, David. Tongues of Fire: the explosion of protestantism in Latin America. Oxford: Blackwell, 1990.

MCDONALD, Robert. An Investigation of Innovation in Nonprofit Organizations: The Role of Organizational Mission. Nonprofit and Voluntary Sector Quarterly, v. 36, n. 2, p. 256-281, jun. 2007.

PFEFFER, Jeffrey; SALANCIK, Gerald. The External Control of Organizations: A Resource Dependence Perspective. New York: Harper and Row, 1978.

PORTER, Michael E. 1985. Competitive advantage: creating and sustaining superior performance. New York: Free Press. 
PORTER, Michael; Kramer, Mark. La creación de valor compartido. Harvard Business Review, p. 32-49, enero/feb. 2011.

RODRÍGUEZ, Dário; Quezada, Soledad. Cultura en organizaciones del tercer sector chileno. Revista española del tercer sector, n. 6, p. 15-57, mayo/agosto 2007.

RUF, Bernadette et al. An empirical investigation of the relationship between Change in Corporate Social Performance and Financial Performance: A Stakeholder Theory Perspective. Journal of Business Ethics, v. 32, p. 143-156, 2001.

STARK, David. El sentido de la disonancia: Reflexividad e Innovación en Organizaciones. Persona y sociedad, v. XIV, n. 1, p. 11-48, abr. 2010.

STARK, David. The Sense of Dissonance: Accounts of Worth in Economic Life. Princeton: Princeton University Press, 2009.

STEWART, Thomas. La nueva riqueza de las organizaciones: el capital intelectual. Buenos Aires: Granica, 1998.

STRAUSS, Anselm; CORBIN, Juliet. Bases para la investigación cualitativa. Medellín: Ed. Universidad de Antioquia, 2002.

TSOUTSOURA, Margarita. Corporate Social Responsibility and Financial Performance. Center for Responsible Business. Working Paper Series, n. 7, 2004. Disponible en: <http://www.haas.berkeley.edu/responsiblebusiness/documents/200405CenterAnnualReport.pdf>. Acceso en: 24 mayo 2008.

WEBER, Max. La ética protestante y el espíritu del capitalismo. Madrid: Sarpe, 1984.

ZADEK, Simon. El camino hacia la responsabilidad corporativa. Harvard Business Review, v. 83, n. 8, p. 54-63, agosto 2005.

ZADEK, Simon. The Civil Corporation: The New Economy of Corporate Citizenship. London, Earthscan: 2007. 